\title{
Reconocimiento de influenza-A como etiología de síndrome febril e insuficiencia respiratoria en adultos hospitalizados durante brote en la comunidad
}

\author{
Ricardo Rabagliati $\mathbf{B}^{1}$, Rosana Benítez $\mathbf{G}^{2}$, \\ Alicia Fernández $\mathrm{M}^{2}$, Pablo $\mathrm{G}$ aete $\mathrm{G}^{3}$, \\ Ana María G uzmán D4, Patricia García C4, \\ Marcela Ferrés $\mathrm{G}^{5}$, Carlos Pérez $\mathrm{C}^{1}$, Jaime Labarca $\mathrm{L}^{1}$. \\ Influenza-A as etiology of fever and \\ respiratory insufficiency in adults \\ hospitalized during an outbreak in Chile
}

\begin{abstract}
Background: Influenza-A (IA) occurs every winter, is mostly observed among outpatients. Aim: To describe the clinical and epidemiological characteristics of cases that required hospital admission during an outbreak in Chile in 1999. Patients and methods: Adults subjects, with Influenza A confirmed by antigen detection test, hospitalized in the clinical hospital of the «ospital Clínico de la Universidad Católica de Chile»between May and June, with fever or respiratory symptoms were studied. A special record was designed to register clinical, microbiological and therapeutic data. Results: Fifty five cases, 26 males, aged 15 to 91 years, were studied. Eighty four percent had chronic concomitant diseases and 9.1\% were immunosuppressed. Clinical findings were fever in $87.3 \%$, asthenia in $83.6 \%$, cough in $93.6 \%$, abnormal pulmonary signs in $69 \%$, an elevated C-reactive protein (mean value of $11.6 \pm 7.1 \mathrm{mg} / \mathrm{dL}$ ) and acute respiratory insufficiency in $54.5 \%$. Cases were isolated in cohort or individual rooms and $38.2 \%$ were admitted to intensive or intermediate care units. Amantadine was prescribed to 52 patients and was well tolerated. Thirty three percent of cases developed pneumonia. These subjects were older, had more dyspnea and respiratory insufficiency than patients without pneumonia. Conclusions: IA should be borne in mind when dealing with hospitalized adults, during epidemic outbreaks in the community. The clinical picture can resemble a serious bacterial infection. An early diagnosis allows the use of specific treatments, to decrease the risk of nosocomial spread and to avoid unnecessary use of antibiotics (Rev Méd Chile 2004; 132: 317-24).
\end{abstract}

(Key Words: Influenza A virus, human; Pneumonia, viral; Respiratory distress syndrome, adult)

Recibido el 29 de agosto, 2003. Aceptado en versión corregida el 11 de diciembre, 2003.

${ }^{1}$ Departamento de Medicina Interna, Programa de Especialización en ${ }^{2}$ Medicina Interna y ${ }^{3}$ Enfermedades Infecciosas del Adulto, ${ }^{4}$ Unidad Docente Asociada de Laboratorios Clínicos y ${ }^{5}$ Laboratorio de Infectología y Virología Molecular. Facultad de Medicina. Pontificia Universidad Católica de Chile.

Correspondencia a: Dr. Ricardo Rabagliati B. Lira 44. Departamento de Medicina Interna. Santiago. Chile. Fax: 354 3175. E-mail: rabagli@med.puc.cl 
La anfluenza es una enfermedad altamente contagiosa, producida por un virus RNA, familia ortomyxoviridae, del que se conocen los tipos A, B y $\mathrm{C}^{1}$, siendo los dos primeros capaces de provocar enfermedad en humanos. Puede afectar a personas de todas las edades, siendo de mayor gravedad en edades extremas ${ }^{2-4}$ o portadores de enfermedades crónicas ${ }^{5-7}$. Clínicamente la influenza A (IA) se manifiesta con un cuadro autolimitado característico: fiebre elevada de comienzo brusco acompañado de calofríos, astenia marcada, mialgias, cefalea, odinofagia y tos seca.

El virus influenza tipo A fue aislado por primera vez en $1933^{8}$, se clasifica en subtipos de acuerdo a las glicoproteínas de superficie, hemaglutinina (H1-H15) y neuraminidasa (N1-N9), las variaciones antigénicas favorecen el desarrollo de brotes epidémicos cada otoño-invierno en ambos hemisferios; cambios más profundos o la introducción de nuevos subtipos, pueden generar brotes que alcanzan magnitudes de pandemias, 0 muy graves como el descrito en 1997 en Hong Kong por el virus aviano A/H5N1 con mortalidad de $42 \%$ en adultos hospitalizados 9 .

Generalmente la influenza se diagnostica y maneja ambulatoriamente con tratamiento sintomático. No es frecuente sospecharla entre los pacientes adultos que requieren hospitalización por síndrome febril o insuficiencia respiratoria, lo que genera una serie de problemas: hospitalización sin implementar medidas de control de infecciones con los consecuentes riesgos de brotes nosocomiales, prolongación de las hospitalizaciones, uso innecesario de antibióticos y mayores costos $^{10,11}$

El objetivo del presente trabajo fue realizar una descripción clínico-epidemiológica de casos de IA diagnosticados en el Hospital Clínico de la Universidad Católica de Chile (HCUC) en el período epidémico de 1999.

\section{PACIENTES Y MÉTODOS}

El HCUC es un hospital universitario de nivel terciario de atención, ubicado en la ciudad de Santiago de Chile, donde anualmente se hospitalizan aproximadamente 25.000 pacientes adultos; cuenta con 520 camas, de las cuales 77 correspon- den a cuidados intensivos o intermedios de pacientes mayores de 15 años.

Desde la semana epidemiológica $17 \mathrm{del}$ año 1999, el proyecto de vigilancia de virus respiratorios de la ciudad de Santiago realizada por el Laboratorio de Infectología y Virología Molecular de nuestra institución ${ }^{12}$, detectó un importante aumento del número de casos de IA en la comunidad, detectándose simultáneamente 2 adultos con IA en nuestro hospital. Debido a este hecho, se diseñó un estudio de vigilancia de IA que consistió en que durante el período comprendido entre los días 2 de mayo y 26 de junio (correspondientes a las semanas epidemiológicas 18 a 25 de 1999) se consideraron como sospechosos de IA pacientes adultos que ingresaban al HCUC, o que estando hospitalizados por otra causa, presentaban sintomatología respiratoria con o sin fiebre o síndrome febril sin foco evidente. Cada paciente sospechoso fue sometido a un test diagnóstico de determinación de antígenos virales, ya sea inmunofluorescencia directa (IFD), test rápido Directigen ${ }^{\circledR} \mathrm{Flu}$ A (Becton, Dickinson and Company, Shannon, Ireland) 0 ambos, de muestra obtenida por hisopado nasofaríngeo. El examen era solicitado por el médico que evaluaba al paciente: residente del servicio de urgencia o la unidad correspondiente de hospitalización o el equipo de infectología. Aquellos con resultado positivo del test para IA fueron definidos como casos. También fueron incluidos como casos aquellos sujetos con diagnóstico de IA realizado en servicio de urgencia del HCUC, si a criterio del médico residente debían ser hospitalizados.

Por cada caso, un médico del grupo de trabajo evaluaba al paciente en el curso de la hospitalización, registrando en una ficha diseñada para este efecto: edad, sexo, antecedentes médicos, vacunación antiinfluenza, motivo de hospitalización, hipótesis diagnóstica al ingreso; unidad de hospitalización; exámenes de laboratorio generales, microbiológicos; radiografía de tórax; tratamiento antiviral y antibiótico; tiempo de hospitalización y condición de egreso. Las recomendaciones terapéuticas y de aislamiento de cada caso, en pieza individual o cohorte, fueron realizadas por el equipo de Infectología y el Comité de Infecciones Intrahospitaliarias del HCUC. 
Análisis estadístico: Se construyó una base de datos en el programa EPI-INFO 6.04, que a su vez se utilizó para realizar el análisis descriptivo. Los resultados de las variables nominales se expresan en porcentajes y de las numéricas en promedio \pm desviación estándar (DS) e intervalo. En el análisis comparativo de los grupos con y sin complicaciones, se utilizó el t-test de Student para las variables continuas y para las variables discontinuas test $\chi^{2}$ y test de Fisher para números $\leq 5$. Se consideró como diferencia estadísticamente significativa el valor $-\mathrm{p} \leq 0,05$.

\section{RESULTADOS}

De un total de 152 test realizados en 147 pacientes hospitalizados, 48 resultaron positivos para IA y 2 para influenza B. Once pacientes con diagnóstico de IA realizado en el servicio de urgencia fueron hospitalizados e incluidos. Se excluyeron 4 casos por datos incompletos. Finalmente se consideraron para este estudio 55 casos.

La distribución de casos diagnosticados por semana demuestra el rápido aumento del número de casos diagnosticados después de la 1a semana con una lenta disminución desde la $4^{\mathrm{a}}$ en adelante (Figura 1). El promedio \pm DS de la edad del grupo resultó $63,5 \pm 20,5$ años (intervalo de 15 a 91 años), 47,2\% sexo masculino, 83,6\% presentaba alguna enfermedad crónica como antecedente médico relevante, destacando: $26 \%$ enfermedad pulmonar obstructiva crónica (EPOC), 15,2\% insuficiencia cardíaca, 15,2\% diabetes mellitus (DM), además en 5 pacientes existía el antecedente de inmunosupresión (3 en tratamiento esteroidal 0 inmunosupresor, un trasplantado cardíaco y otro paciente con SIDA).

Respecto a la fuente de contagio, 18 pacientes $(32,7 \%)$ reconocieron haber estado en contacto con algún enfermo con síndrome gripal. En 6 casos la adquisición fue intrahospitalaria, en 3 casos se identificó contacto en la misma sala de algún paciente con IA, 2 habían sido visitados por familiares con síndrome gripal y en un caso no quedó clara la fuente. Solamente 6 pacientes $(10,9 \%)$ habían recibido la vacuna antigripal correspondiente.

Dentro de las características clínicas, destacaban fiebre elevada, astenia y tos (Tabla 1). El diagnóstico fue realizado con test rápido en 40 casos y en 16 IFD (un paciente con test rápido negativo e inmunofluorescencia positiva y otro caso resultó con ambos test positivos). El diagnóstico de IA se realizó generalmente dentro de las primeras $24 \mathrm{~h}$ del ingreso o del inicio de los síntomas para el grupo de adquisición nosocomial (0,8 $\pm 1,15$ días; intervalo $0-5)$.

Entre los exámenes de laboratorio cabe destacar la desviación a izquierda ( $\geq 5 \%$ baciliformes) observada en $58 \%$ de los pacientes, linfopenia ( $\leq 10 \%$ de linfocitos) en $45 \%$ de los casos y elevación de la proteína $\mathrm{C}$ reactiva (Tabla 2 ).

En la Tabla 3 se señalan el motivo de hospitalización y la hipótesis diagnóstica al momento del ingreso. Los 6 pacientes que adquirieron IA en el hospital, estaban hospitalizados por: apendicitis aguda, artroplastia de cadera, hipertensión pulmo-

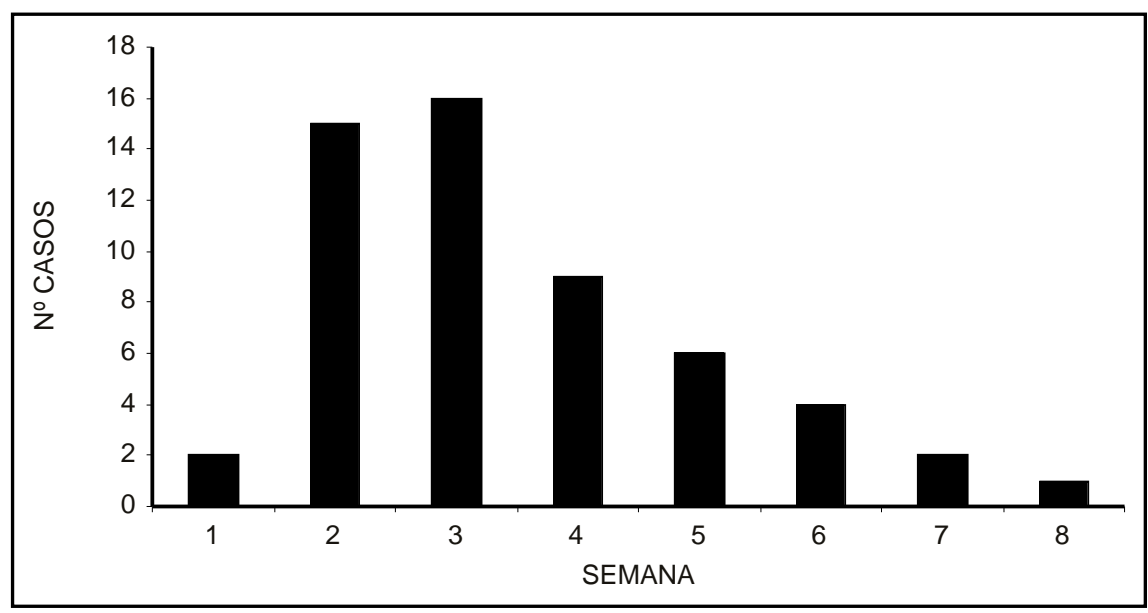

Figura 1. Distribución semanal del número de casos diagnosticados de IA en el período comprendido entre el 2 de mayo al 26 de junio de 1999 (correspondientes a las semanas epidemiológicas 18 a 25). 
Tabla 1. Características del cuadro clínico de 55 pacientes con IA hospitalizados

\begin{tabular}{|lrc|}
\hline & $\mathrm{n}$ & $\%$ \\
\hline Síntomas & & \\
Tos & 53 & 93,6 \\
Fiebre $\left(>38^{\circ} \mathrm{C}\right)$ & 48 & 87,3 \\
Astenia & 46 & 83,6 \\
Expectoración & 39 & 70,9 \\
Calofríos & 37 & 67,2 \\
Mialgias & 37 & 67,2 \\
Disnea & 30 & 54,5 \\
Cefalea & 24 & 43,6 \\
Congestión nasal & 22 & 40,0 \\
Odinofagia & 12 & 21,8 \\
Vómitos & 15 & 27,3 \\
Diarrea & 6 & 10,9 \\
Dolor traqueal & 6 & 10,9 \\
Signos & & \\
Crepitaciones & 35 & 63,6 \\
Espiración prolongada & & \\
$\quad$ y/o sibilancias & 29 & 52,7 \\
Taquicardia* & 23 & 41,8 \\
Polipnea** & 19 & 34,5 \\
\hline
\end{tabular}

* Frecuencia cardíaca $>100$ latidos por min

** Frecuencia respiratoria $>20$ respiraciones por min nar, síndrome nefrótico, tromboembolismo pulmonar y nefrectomía.

El 38,2\% de los pacientes fue hospitalizado en unidades de cuidados intermedios 0 intensivos, debido especialmente a insuficiencia respiratoria. Un tercio de los casos requirió $\mathrm{FiO}_{2}>30 \%$ y 6 casos requirieron conexión a ventilación mecánica.

En 18 (32,7\%) pacientes se diagnosticó neumonía sobre la base del compromiso pulmonar

\section{Tabla 2. Características de principales exámenes de laboratorio de 55 sujetos hospitalizados con diagnóstico de IA}

\begin{tabular}{|lcc|}
\hline & Promedio \pm DS & Intervalo \\
\hline $\begin{array}{l}\text { Recuento de leucocitos } \\
\text { (cel/mm } \text { m }^{3}\end{array}$ & $9.788 \pm 4.677$ & $(4.800-28.100)$ \\
Desviación a izquierda & & \\
$\quad$ [n (\%)] & $32(58)$ & \\
Lifopenia [n (\%)] & $25(45,5)$ & \\
VHS (mm/hora) & $32,9 \pm 27$ & $(3-112)$ \\
PCR* (mg/dL) & $11,6 \pm 7,1$ & $(1-27)$ \\
$\mathrm{PaO}_{2} / \mathrm{FiO}_{2}$ & $298 \pm 81$ & $(120-528)$ \\
\hline
\end{tabular}

*valor normal $0-0,9 \mathrm{mg} / \mathrm{dL}$

Tabla 3. M otivo de hospitalización y diagnóstico de ingreso de 49 pacientes con IA adquirida en la comunidad

\begin{tabular}{|lcc|}
\hline & $\mathrm{n}$ & $\%$ \\
\hline Motivo de hospitalización & & \\
Insuficiencia respiratoria aguda o crónica reagudizada & 18 & 36,7 \\
$\quad$ (incluye EPOC descompensado) & 16 & 32,6 \\
Síndrome febril & 10 & 20,4 \\
Neumonía o sospecha de neumonía & 3 & 6,1 \\
Arritmia, angor inestable, infarto al miocardio & 1 & 2,0 \\
DM descompensada & 1 & 2,0 \\
Daño hepático crónico descompensado & & \\
Diagnóstico de ingreso & 25 & 51,0 \\
Neumonía o sospecha de neumonía & 11 & 22,4 \\
IA & 4 & 8,2 \\
Síndrome febril & & \\
Insuficiencia respiratoria aguda o crónica reagudizada & 5 & 10,2 \\
$\quad$ (incluye EPOC descompensado) & 3 & 6,1 \\
Arritmia, angor inestable, infarto al miocardio & 1 & 2,0 \\
DM descompensada & & \\
\hline
\end{tabular}


observado en la radiografía de tórax, condensación en 15 pacientes e infiltrado intersticial en 3, de un total de 46 radiografías de tórax. El cultivo de expectoración fue positivo en 2 casos, $\mathrm{S}$ pneumoniae en uno y $\mathrm{H}$ influenzae en otro, ningún hemocultivo resultó positivo. No se realizó estudio serológico de atípicos.

Se administró amantadina como terapia antiviral estándar en $52(94,5 \%)$ casos ajustado por edad y función renal de acuerdo a las recomendaciones habituales para este medicamento, en general su uso fue bien tolerado, con excepción de un paciente, que había recibido simultáneamente sulfas, que desarrolló alteración cualitativa de conciencia. El 57\% de los pacientes se hizo afebril a las $24 \mathrm{~h}$ de iniciada la amantadina. El $65 \%$ de los pacientes recibió adicionalmente antibióticos por vía intravenosa y luego oral.
Al comparar los grupos de sujetos diagnosticados con neumonía respecto a aquellos sin neumonía se observaron algunas diferencias. En particular el grupo con neumonía era significativamente de edad más avanzada, presentaba con mayor frecuencia disnea y alteraciones del examen pulmonar (crepitaciones, espiración prolongada o sibilancias) junto a una menor relación $\mathrm{PaO}_{2} / \mathrm{FiO}_{2}$. También presentaban más elevadas la VHS y PCR y mayor frecuencia de desviación a izquierda, pero sin alcanzar diferencia estadísticamente significativa (Tabla 4).

Otras complicaciones observadas fueron: en 1 paciente rabdomiolisis leve y en 12 casos alteración de conciencia (no relacionado con el uso de amantadina).

El promedio de la estadía hospitalaria fue 7,4 \pm 6,5 días (1 a 40 días). Una paciente (1,8\%) falleció, era cardiópata y se diagnosticó neumonía.

Tabla 4. Comparación de características clínico-epidemiológicas de sujetos con IA en que se diagnosticó neumonía (18 pacientes) con aquellos en que no se diagnosticó neumonía (37 pacientes). D efinición de neumonía según el compromiso pulmonar observado en la radiografía de tórax

\begin{tabular}{|c|c|c|c|}
\hline & $\begin{array}{c}\text { Sin neumonía } \\
\text { promedio } \pm \text { DS/n (\%) }\end{array}$ & $\begin{array}{c}\text { Con neumonía } \\
\text { promedio } \pm \text { DS/n (\%) }\end{array}$ & $\mathrm{p}$ \\
\hline Edad (años)* & $56,8 \pm 20,9$ & $77,4 \pm 105$ & 0,03 \\
\hline Sexo masculino & $16(43)$ & $10(55,5)$ & 0,4 \\
\hline Enfermedad crónica** & $28(75,7)$ & $18(100)$ & 0,06 \\
\hline Días pre-hospitalización & $2,5 \pm 1,5$ & $3,8 \pm 2,3$ & 0,15 \\
\hline Fiebre $\left(>38^{\circ} \mathrm{C}\right)$ & $32(86,5)$ & $16(88,9)$ & 0,9 \\
\hline Calofríos & $27(73)$ & $10(55,6)$ & 0,2 \\
\hline Tos & $36(97,3)$ & $17(94,4)$ & 0,9 \\
\hline Expectoración & $25(67,6)$ & $14(77,8)$ & 0,4 \\
\hline Disnea* & $13(35,1)$ & $17(94,4)$ & 0,00003 \\
\hline Uso de antibióticos pre hospitalización & $8(21,6)$ & $4(22,2)$ & 0,8 \\
\hline Frecuencia respiratoria (resp/min) & $24,4 \pm 6,4$ & $28,1 \pm 7,3$ & 0,06 \\
\hline Crepitaciones* & $19(51,4)$ & $16(88,9)$ & 0,007 \\
\hline Espiración prolongada y/o sibilancias* & $13(35,1)$ & $16(88,9)$ & 0,0002 \\
\hline Recuento leucocitos (cel/ $\mathrm{mm}^{3}$ ) & $8,89 \pm 3,72$ & $11,38 \pm 5,79$ & 0,2 \\
\hline Desviación a izquierda & $19(51,4)$ & $13(72,2)$ & 0,14 \\
\hline VHS (mm/hora) & $26,4 \pm 21,5$ & $44,3 \pm 32,3$ & 0,15 \\
\hline $\mathrm{PCR}^{* * * *}(\mathrm{mg} / \mathrm{dl})$ & $8,6 \pm 3,9$ & $16,5 \pm 8,4$ & 0,12 \\
\hline $\mathrm{PaO}_{2} / \mathrm{FiO}_{2} *$ & $323,8 \pm 67,9$ & $244,7 \pm 81,6$ & 0,0003 \\
\hline Tiempo hospitalización (días) & $5,3 \pm 3,2$ & $11,5 \pm 9$ & 0,1 \\
\hline
\end{tabular}

*variables con diferencia estadísticamente significativa (valor-p <0,05).

* EPOC, Insuficiencia cardíaca, DM, inmunosupresión.

*** PCR medida en 16 pacientes sin neumonía y en 10 pacientes con neumonía. 


\section{DisCUSIÓN}

El elevado número de casos de IA diagnosticados en nuestro hospital durante las 8 semanas de estudio, fue reflejo del comportamiento del virus influenza en la comunidad. La oportuna información de la vigilancia aportada por el proyecto de vigilancia de virus respiratorios de nuestra institución ${ }^{12}$ fue fundamental para iniciar la búsqueda activa de casos hospitalizados.

Como era de esperar la mayoría de nuestros casos era de la tercera edad $(61,8 \%$ edad $\geq 65$ años) y el $83,6 \%$ presentaba algún antecedente médico relevante, en estas circunstancias el cuadro clínico de fiebre elevada, polipnea, insuficiencia respiratoria y desviación a izquierda sugería una infección bacteriana grave. De nuestra experiencia, es importante recalcar que la IA en el grupo de adultos mayores el cuadro clínico puede sugerir fuertemente neumonía bacteriana o ser atípico para una infección viral, por lo que en este grupo de pacientes puede resultar particularmente difícil el proceso diagnóstico. Un alto grado de sospecha y el conocimiento de la situación epidemiológica del momento, son elementos claves en el diagnóstico de IA.

Los resultados de nuestro trabajo ponen de manifiesto la importancia del diagnóstico de la infección por virus respiratorios. En adultos está descrita la IA como factor descompensante de enfermos respiratorios crónicos o cardiópatas ${ }^{5}$ y como causante de fiebre en pacientes inmunocomprometidos $^{6,7}$; recientemente también se ha reconocido la importancia del virus respiratorio sincicial (VRS) ${ }^{3,13}$. En un estudio prospectivo que evaluó la etiología viral en pacientes mayores de 65 años hospitalizados por neumonía, descompensación de patología cardiorrespiratoria o síndrome gripal, se identificó IA en $11 \%$ y VRS en $10 \% 3$.

Dentro de las complicaciones existen frecuentes alteraciones de la función pulmonar: disfunción de la vía aérea y alteraciones del intercambio gaseoso que sugiere la invasión viral del tracto respiratorio bajo. Respecto a la primera, la hiperreactividad bronquial se reconoce como un hecho común y autolimitado en la IA no neumónica 14,15 , cabe destacar que cerca de $60 \%$ de nuestros pacientes desarrolló al menos signología de obstrucción bronquial. La insuficiencia respiratoria observada en nuestra serie puede ser multifactorial: compromiso pulmonar viral, obstrucción bronquial, infección bacteriana asociada, insuficiencia cardíaca y EPOC descompensado. La importancia de esta complicación queda reflejada en el tercio de casos que requirió $\mathrm{FiO}_{2}>30 \%$ y 10,9\% ventilación mecánica; incluso, en la literatura se reportan casos de IA que han requerido apoyo de membrana de oxigenación extracorpórea debido a insuficiencia respiratoria grave ${ }^{16}$.

Entre otras complicaciones descritas para IA, solamente observamos un caso de rabdomiolisis leve, existiendo casos descritos de rabdomiolisis con falla renal aguda ${ }^{17}$. Las alteraciones de conciencia observadas en $21,8 \%$ pueden estar con relación a la edad avanzada de muchos de nuestros pacientes; en la literatura también se describen casos de encefalopatía o encefalitis con demostración de virus IA en líquido cefalorraquídeo ${ }^{18}$. No observamos complicaciones como miocarditis, pericarditis, o síndrome de shock tóxico asociado a infección por Staphylococcus post IA ${ }^{19,20}$.

Es útil disponer de métodos de detección rápida de IA, permiten confirmar el diagnóstico e implementar las medidas terapéuticas y epidemiológicas respectivas. La sensibilidad descrita para el test rápido Directigen $\mathrm{FluA}^{\circledR}$ es 67 a $96 \%$ con una especificidad de 88 a $97 \% 21$ y para inmunofluorescencia la sensibilidad y especificidad es sobre $90 \% 22$. Existen otros métodos como serologías, reacción de polimerasa en cadena, y cultivo viral, pero resultan lentos o costosos ${ }^{21}$.

Respecto al compromiso radiológico pulmonar, es muy difícil señalar categóricamente si éste era exclusivamente debido a compromiso viral o bacteriano. A pesar que el estudio estándar destinado a descartar neumonía bacteriana con hemocultivos y estudio de expectoración, es de bajo rendimiento ${ }^{23}$ y que no disponemos de estudio serológico de atípicos, los resultados negativos de hemocultivos y el resultado positivo del cultivo de expectoración en 2 sujetos solamente, hace planteable que el compromiso pulmonar pueda haber sido de naturaleza viral en algunos de nuestros pacientes. Tampoco se puede descartar que algunos hayan presentado co-infección viral y bacteriana en forma simultánea o secuencial.

El estudio no fue destinado a evaluar la respuesta a la amantadina, pero cabe señalar que 
junto a la rimantadina está descrita su efectividad en la profilaxis y tratamiento de la $\mathrm{IA}^{24}$. Su mecanismo de acción es la interferencia con la replicación viral al bloquear la función de la proteína M2, esencial para descubrir el virus luego de su entrada a la célula ${ }^{24}$. Existen nuevos antivirales que actúan como inhibidores de la neurominidasa: zanimavir y oseltamivir, que ofrecen mejores resultados que amantadina, incluyendo acción sobre virus influenza $B$, pero a mayor costo ${ }^{25}$.

No podemos precisar la causa del fracaso de la vacuna en 6 casos, debido a que desconocemos el momento en que la recibieron. Se debe considerar que la eficacia de las vacunas parenterales con virus inactivado es de $68 \% 26$. En sujetos mayores de 64 años está descrito su efecto con reducción de hospitalización por neumonía e influenza de 48-57\%, de cualquier condición respiratoria de 27 $39 \%$ y disminución de 37\% de hospitalización por insuficiencia cardíaca al evaluar 3 estaciones de influenza ${ }^{27}$. Son promisorias las investigaciones con una nueva vacuna de administración intranasal, que provoca respuesta inmune mucosa, además de respuesta sistémica, los estudios realizados han establecido la seguridad, inmunogenicidad y efectividad de este tipo de vacunas ${ }^{28,29}$.

\section{REFERENCIAS}

1. Miuer M. Viral taxonomy. Clin Infect Dis 1999; 29: 731-3.

2. Thompson WW, Shay DK, Weintraub E, Brammer L, Cox N, ANDERson LJ ET AL. Mortality associated with influenza and respiratory syncytial virus in the United States. JAMA 2003; 289: 179-86.

3. FaLSEY AR, CunNingham CK, Barker WH, Kouidas RW, Yuen JB, Menegus M et AL. Respiratory Syncytial virus and Influenza A infections in the hospitalized elderly. J Infect Dis 1995; 172: 38994.

4. Walsh EE, Cox C, Falsey AR. Clinical features of influenza A virus infection in older hospitalized persons. J Am Geriatric Soc 2002; 50: 1498-503.

5. Walsh EE, Falsey AR, Hennessey PA. Respiratory syncitial and other virus infection in persons with chronic cardiopulmonary disease. Am J Respir Crit Care Med 1999; 160: 791-5.
La infección intrahospitalaria es otro aspecto importante a destacar, el desarrollo de brotes nosocomiales de influenza es un hecho muy temido, dada la evolución de la enfermedad, la eventual prolongación de la hospitalización y mayores $\operatorname{costos}^{10}$. Por esto resulta fundamental la vigilancia y detección de nuevos casos para su oportuno aislamiento. Otras recomendaciones son la suspensión de visitas de familiares y el programa de vacunación del personal de salud 10,11,30-32.

En conclusión, el reconocimiento de los brotes de IA en la comunidad, facilitado por una vigilancia epidemiológica adecuada, que entregue información oportuna, constituye un elemento central para generar un estado de alerta que lleva al equipo de salud a tener una actitud activa para sospechar y confirmar casos de IA en aquellos pacientes que cursan un cuadro febril agudo, con o sin síntomas respiratorios, con o sin insuficiencia respiratoria, permitiendo realizar un diagnóstico precoz, iniciar las medidas terapéuticas específicas, y en caso de hospitalización implementar las medidas oportunas de control de infecciones intrahospitalarias.

6. Martino R, Rámila E, Rabelia N, Muñoz JM, Peyret M, Portos JM ET AL. Respiratory virus infections in adults with hematologic malignancies: a prospective study. Clin Infect Dis 2003; 36: 1-8.

7. Fine $\mathrm{AD}$, Bridges $\mathrm{CB}, \mathrm{De}$ Guzmán AM, Glover L, Zeler B, Wong SJ et al. Influenza A among patients with human immunodeficiency virus: an outbreak of infection at a residencial facility in New York City. Clin Infect Dis 2001; 32: 1784-91.

8. Smith W, Andrewes CH, Laidlaw PP. A virus obtained from influenza patients. Lancet 1933; 2: 66-8.

9. Yuen KY, Chan PK, Peiris M, Tsang DN, Que TL, Shortridge KF et aL. Clinical features and rapid viral diagnosis of human disease associated with avian influenza A H5N1 virus. Lancet 1998; 351: 467-71.

10. Salgado CD, Farr BM, HaLl KK, Hayden FG. Influenza in the acute hospital setting. Lancet Infect Dis 2002; 2: 145-55. 
11. Weingarten S, Friediander M, Rascon D, Ault M, Morgan M, MEYeR RD. Influenza surveillance in an acute care hospital. Arch Intern Med 1988; 148: 113-6.

12. Proyecto Vigilancia Virus Respiratorios. Identificación virus circulantes 1999. Laboratorio de Infectología y Biología Molecular. Programa de Enfermedades Infecciosas. Pontificia Universidad Católica de Chile. Facultad de Medicina. Centro de Investigaciones Médicas. URL disponible en: http:// contacto.med.puc.cl/virus/virus_respiratorios/ bienvenida.html»

13. Dowell SF, Anderson LJ, Gary HE JR, ERdman DD, PloufFe JF, FiLe TM JR et aL. Respiratory syncicial virus is an important cause of community-acquired lower respiratory infection among hospitalized adults. J Infect Dis 1996; 174: 456-62.

14. Lttie JW, Hall WJ, Douglas RG JR, Mudholkar GS, Speers DM, Patel K. Airway hyperrreactivity and peripheral airway dysfunction in influenza A infection. Am Rev Respir Dis 1978; 118: 295-303.

15. Minor TE, Dick EC, Baker JW, Ouelette JJ, Cohen $\mathrm{M}$, REED CE. Rhinovirus and influenza type an infectious as precipitants of asma. Am Rev Respir Dis 1976; 113: 149-53.

16. Lefrak EA, Stevens PM, Pitha J, Balsinger E, Noon GP, MAYOR HD. Extracorporeal membrane oxigenation for fulminant influenza pneumonia. Chest 1974; 66: 385-8.

17. Minow R, Sherwood G, Johnson L et al. Myoglobinuria associated with influenza A infection. Ann Inter Med 1974; 80: 359-61.

18. Fujimoto S, Kobayashi M, Uemura O, Iwasa M, Ando T, КатоH T ET AL. PCR on cerebrospinal fluid to show influenza associated acute encephalopaty or encephalitis. Lancet 1998; 352: 873-5.

19. MacDonald KL, Osterholm MT, Hedberg CW, Schrock CG, Peterson GF, Jentzen JM et al. Toxic shock syndrome a newly recognized complication of influenza and influenza like illness. JAMA 1987; 257: 1053-8.

20. Sperber S, Francis B. Toxic shock syndrome during an influenza outbreak. JAMA 1987; 257: 1086-7.

21. Cox N, Subbarao K. Influenza. Lancet 1999; 354: 1277-82.

22. Leonardi GP, Leib H, Birkhead GS, Smith C, CosteLO P, ConRon W. Comparision of rapid detection methods for influenza A virus and their value in health care management of institutionalized geriatric patients. J Clin Microbiol 1994; 32: 70-4.

23. Saldías F, Mardónez JM, Marchesse M, Viviani $P$, Farías G, Díaz A. Neumonía adquirida en la comunidad en el adulto hospitalizado. Cuadro clínico y factores pronósticos. Rev Méd Chile 2002; 130: 1373-82.

24. Jefrerson TO, Demichel V, Deeks JJ, Rivetti D. Amantadine and rimantadine for preventing and treating influenza A in adults. Cochrane Database Syst Rev 2002; (3): CD001169.

25. Sмтtн KJ, RobeRTs MS. Cost-effectiveness of newer treatment strategies for influenza. Am J Med 2002; 113: 300-7.

26. Demichel V, Rivetti D, Deeks JJ, JefFerson tO. Vaccines for preventing influenza in healthy adults. Cochrane Database Syst Rev 2001; (4): CD001269.

27. Nichol KL, Margous KL, Wuorenma J, Von SternBERG T. The efficacy and cost effectiveness of vaccination against influenza among elderly persons living in the community. N Engl J Med 1994; 331: 778-84.

28. Bernstein DI, Yan L, TReanor J, Medelman PM, BeLSHe R. Cold adapted, trivalent, Influenza Vaccine Study Group. Pediatr Infectious Dis J 2003; 22: 28-34.

29. Beyer WE, Palache AM, De Jong JC, Osterhaus AD. Cold Adapted live influenza vaccine versus inactivated vaccine: systemic vaccine reactions, local and systemic response and vaccine efficacy. A meta-analysis. Vaccine 2002; 20: 1340-53.

30. Weinstock DM, Eagan J, Malak SA, Rogers M, WaLace H, KieHn TE et al. Control of influenza A on a bone marrow transplant unit. Infect Control Hosp Epidemiol 2000; 21: 730-2.

31. Pachucki CT, Pappas SA, Fuller GF, Krause SL, Lentino JR, SchaAfF DM. Influenza A among hospital personnel and patients. Implications for recognition, prevention, and control. Arch Intern Med 1989; 149: 77-80.

32. Carman WF, Elder AG, Wallace la, McAulay K, WALKer A, MuRRay GD et al. Effects of influenza vaccination of health care workers on mortality of elderly people in long term care: a randomized controlled trial. Lancet 2000; 355: 93-7. 